\title{
Complex Dissipating Energy Flow Method for Forced Oscillation Source Location
}

This paper was downloaded from TechRxiv (https://www.techrxiv.org).

\section{LICENSE}

CC BY 4.0

SUBMISSION DATE / POSTED DATE

18-02-2022 / 23-02-2022

\section{CITATION}

Gill Estevez, Pablo; Marchi, Pablo; Galarza, Cecilia; Elizondo, Marcelo (2022): Complex Dissipating Energy Flow Method for Forced Oscillation Source Location. TechRxiv. Preprint. https://doi.org/10.36227/techrxiv.19196327.v1

DOI

10.36227/techrxiv.19196327.v1 


\title{
Complex Dissipating Energy Flow Method for Forced Oscillation Source Location
}

\author{
Pablo Gill Estevez, Pablo Marchi, Cecilia Galarza and Marcelo Elizondo
}

\begin{abstract}
The original Dissipating Energy Flow (DEF) method for locating sources of forced oscillations (FOs) is based on an energy function that neglects transference conductances. In this paper we propose a Complex DEF (CDEF) whose projection onto a specific direction, defined from a representative $R / X$ ratio of the system branches, is used to trace sources of FO. It is shown by simulated cases that the CDEF has a good performance in cases where the original DEF fails.
\end{abstract}

Index Terms-Forced oscillations source location, phasor measurement unit (PMU), energy functions, dissipating energy flow.

\section{INTRODUCTION}

$\mathbf{T}$ HE Dissipating Energy Flow (DEF) method [1] for locating sources of forced oscillations (FOs) is based on the conventional energy function of a power system [2] that assumes that the network is composed purely of transference susceptances, neglecting transference conductances. Although $R / X$ ratio is small in high voltage transmission systems, neglecting transference conductances can cause the conventional DEF method to be inaccurate in some circumstances [3], [4].

Energy functions constructed by the complex integral approach can be divided into its real and imaginary parts [5]. The latter provides the conventional energy function while the former provides a second-kind energy function. It has been shown that a series of energy functions can be generated by taking the weighted sum of the conventional energy function and the second-kind one [6]. By taking appropriate weights, a well-defined energy function can be derived to reflect the transfer conductance when the system has uniform $R / X$ ratio for all system branches. This approach was developed in [7], and similar energy functions for uniform $R / X$ ratio were also previously proposed in [8], [9]. In [6], it was generalized so as not to restrict internal $R / X$ ratio of generators.

In this work we propose an extension of the DEF method, which we call Complex DEF (CDEF) method. The CDEF is defined from the complex energy function [5]. Justified from the energy function [6], we use the scalar projection of CDEF on a specific direction (defined from a representative $R / X$ ratio of the system branches) to trace sources of FO.

\section{ENERGy FunCtion BASED EN COMPLEX INTEGRAL APPROACH}

The complex integral approach for the definition of energy function was defined in [5] by considering the complex integral

P. Gill Estevez, P. Marchi, and C. Galarza are with the School of Engineering, Universidad de Buenos Aires and the CSC-CONICET, Argentina. M. Elizondo works with Pacific Northwest National Laboratory, Seattle, USA. (e-mail: pgill@fi.uba.ar, pmarchi@fi.uba.ar, cgalar@fi.uba.ar, marcelo.elizondo@pnnl.gov) relationships of bus current equations. For a network with $N$ buses and $m$ generators, the following complex energy function can be defined

$$
\mathbf{W}=\int \sum_{i=1}^{N} \sum_{k=1}^{N} \mathbf{Y}_{\mathbf{i k}}^{*} \mathbf{V}_{\mathbf{k}}^{*} \mathbf{d} \mathbf{V}_{\mathbf{i}}-\sum_{i=1}^{m} \mathbf{I}_{\mathbf{G i}}^{*} \mathbf{d} \mathbf{V}_{\mathbf{i}}+\sum_{i=1}^{N} \mathbf{I}_{\mathbf{L i}}^{*} \mathbf{d} \mathbf{V}_{\mathbf{i}}=\mathbf{0}
$$

Where $\mathbf{Y}_{\mathbf{i k}}=G_{i k}+j B_{i k}$ are the coefficients of the bus admittance matrix, $\mathbf{I}_{\mathbf{G}}$ and $\mathbf{I}_{\mathbf{L}}$ are the current injections of generation and load buses. The integration limits are determine by the trajectory of the system variables between two operation points. On the other hand, by substitution the following relationships: $\mathbf{V}_{\mathbf{i}}=V_{i} e^{j \theta_{i}}, \mathbf{I}_{\mathbf{G i}}^{*}=\left(P_{G i}+j Q_{G i}\right) / V_{i} e^{-j \theta_{i}}$, $\mathbf{I}_{\mathbf{L i}}^{*}=\left(P_{L i}+j Q_{L i}\right) / V_{i} e^{-j \theta_{i}}, \mathbf{d V}_{\mathbf{i}}=e^{j \theta_{i}} d V_{i}+j V_{i} e^{j \theta_{i}} d \theta_{i}$ into (1), we can derive the imaginary part $W_{I M A G}=\operatorname{Im}(\mathbf{W})$ and real part $W_{R E A L}=\operatorname{Re}(\mathbf{W})[6]$ :

$$
\begin{gathered}
W_{I M A G}=-\left[\frac{1}{2} \sum_{i=1}^{N} B_{i i} V_{i}^{2}+\frac{1}{2} \sum_{i=1}^{N} \sum_{k \neq i}^{N} B_{i k} V_{i} V_{k} \cos \theta_{i k}\right]+ \\
+\sum_{i=1}^{N} \sum_{k=1}^{N} \int G_{i k}\left(V_{k} \sin \theta_{i k} d V_{i}+V_{i} V_{k} \cos \theta_{i k} d \theta_{i}\right)- \\
-\sum_{i=1}^{m} \int\left(P_{G i} d \theta_{i}+\frac{Q_{G i}}{V_{i}} d V_{i}\right)+\sum_{i=1}^{N} \int\left(P_{L i} d \theta_{i}+\frac{Q_{L i}}{V_{i}} d V_{i}\right)-C=0 \\
W_{R E A L}=\left[\frac{1}{2} \sum_{i=1}^{N} G_{i i} V_{i}^{2}+\frac{1}{2} \sum_{i=1}^{N} \sum_{k \neq i}^{N} G_{i k} V_{i} V_{k} \cos \theta_{i k}\right]+ \\
+\sum_{i=1}^{N} \sum_{k=1}^{N} \int B_{i k}\left(V_{k} \sin \theta_{i k} d V_{i}+V_{i} V_{k} \cos \theta_{i k} d \theta_{i}\right)- \\
-\sum_{i=1}^{m} \int\left(\frac{P_{G i}}{V_{i}} d V_{i}-Q_{G i} d \theta_{i}\right)+\sum_{i=1}^{N} \int\left(\frac{P_{L i}}{V_{i}} d V_{i}-Q_{L i} d \theta_{i}\right)-C^{\prime}=0
\end{gathered}
$$

where $C$ and $C^{\prime}$ are constants that depend on the initial conditions. $W_{I M A G}$ is typically used to define a conventional energy function [10]. However, if the transfer conductances $G_{i k}$ are not neglected, then the associated terms remain pathdependent, without being able to establish a well-defined energy function.

If we assume a uniform $R / X$ ratio for all branches in the system $\left(K=R_{i k} / X_{i k}\right)$, then the transfer conductance and susceptances would have the following relationships [6]:

$$
B_{i k}=\frac{1}{X_{i k}} \frac{-1}{\left(K^{2}+1\right)} ; G_{i k}=-K B_{i k}=\frac{1}{X_{i k}} \frac{K}{\left(K^{2}+1\right)}
$$

The proportional relationship between $B_{i k}$ and $G_{i k}$ allows us to express path-dependent integration term related to transfer 
conductances of (2) from (3). In this way, an energy function for a system with fixed $R / X$ ratio can be defined [6]:

$$
\begin{aligned}
& W_{E X T}=\frac{1}{\sqrt{1+K^{2}}}\left[W_{I M A G}+K W_{R E A L}\right]= \\
& =\frac{1}{\sqrt{1+K^{2}}}\left[-\frac{1}{2} \sum_{i=1}^{N} B_{i i 0} V_{i}^{2}-\frac{1}{2} \sum_{i=1}^{N} \sum_{k \neq i}^{N} B_{i k 0} V_{i} V_{k} \cos \theta_{i k}-\right. \\
& \quad-\sum_{i=1}^{m} \int\left(P_{G i} d \theta_{i}+\frac{Q_{G i}}{V_{i}} d V_{i}+K\left(-Q_{G i} d \theta_{i}+\frac{P_{G i}}{V_{i}} d V_{i}\right)\right)+ \\
& \left.+\sum_{i=1}^{N} \int\left(P_{L i} d \theta_{i}+\frac{Q_{L i}}{V_{i}} d V_{i}+K\left(-Q_{L i} d \theta_{i}+\frac{P_{L i}}{V_{i}} d V_{i}\right)\right)\right]-C^{\prime \prime}=0
\end{aligned}
$$

where $B_{i k 0}=B_{i k}-K G_{i k}=1 / X_{i k}, B_{i i 0}=-\sum 1 / X_{i k}$ and $C^{\prime \prime}$ is a constant that depends on the initial conditions.

\section{Proposed CoMplex DEF}

Based on the complex energy function (2)(3), we propose the definition of the Complex DEF (CDEF) as:

$$
\begin{aligned}
\mathbf{W}_{\mathbf{c}}^{\mathbf{i k}} & =j \int\left(\Delta P_{i k} d \Delta \theta_{i}+\frac{\Delta Q_{i k}}{V_{i}} d \Delta V_{i}\right)+ \\
& +\int\left(-\Delta Q_{i k} d \Delta \theta_{i}+\frac{\Delta P_{i k}}{V_{i}} d \Delta V_{i}\right)
\end{aligned}
$$

The path dependent terms of (5) can be associated to the projection of CDEF onto the direction defined by $K+j$, which we call Projected DEF (PDEF):

$$
\begin{aligned}
W_{d}^{i k} & =\frac{1}{\sqrt{1+K^{2}}}\left[\int\left(\Delta P_{i k} d \Delta \theta_{i}+\frac{\Delta Q_{i k}}{V_{i}} d \Delta V_{i}\right)+\right. \\
& \left.+K \int\left(-\Delta Q_{i k} d \Delta \theta_{i}+\frac{\Delta P_{i k}}{V_{i}} d \Delta V_{i}\right)\right]
\end{aligned}
$$

where $K$ is the $R / X$ ratio representative of the branches of the transmission system under analysis, $P_{i k}$ and $Q_{i k}$ are the active and reactive power flows in branch $i k, \theta_{i}$ is the voltage angle, and $V_{i}$ is voltage magnitude of from bus. $\Delta$ indicates filtered magnitudes corresponding to the oscillatory frequency component. The integration limits are determined from the instant when sustained oscillations have significant magnitude. The value and sign of the rate of change of $W_{d}^{i k}$ indicates the direction of the FO source location relative to the branch $i k$. Positive rate of change of $W_{d}^{i k}$ means the source is located behind bus $i$, and a negative value means the source is located behind bus $k$ or branch $i k$ is the source [11]. Note that for $K=0,(7)$ is identical to the original DEF [1] [12].

\section{NumericAl Results}

\section{A. Kundur's Two-Area System}

We use the version of Kundur's two-area system [13] with the original PSS settings available in [14]. All transmission lines represented in the model have $R / X=0.1$, therefore for the calculation of the PDEF we use $K=0.1$ in this case. Loads are represented with $40 \% \mathrm{Z}, 30 \% \mathrm{I}$ and $30 \% \mathrm{P}$.

In the first four cases, FO is applied in each generator, one at a time, in the field voltage $E_{f d}$. The amplitude of the FO is 0.1 pu and the frequency is $0.3 \mathrm{~Hz}$. For the first case, Fig. 1 shows that the original DEF incorrectly classifies G2 as a source (positive rate of change of DEF), while the PDEF correctly classifies G1 as a source. Fig. 2 shows polar plots at $t=60 \mathrm{~s}$ for each case where the CDEF in each generator is represented. The shaded zone of the plot is the region where the PDEF is positive (where a generator would be classified as a source). On the other hand, the original DEF can be calculated as the projection of the CDEF onto the imaginary axis. In in the four cases, the original DEF fails and the PDEF correctly classifies the FO sources.
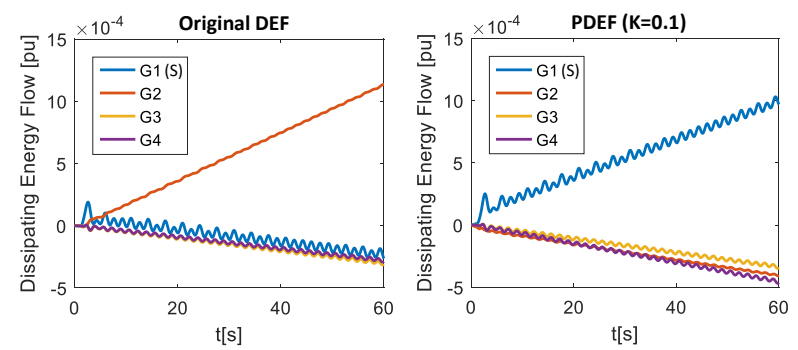

Fig. 1. Case 1: FO in $E_{f d}$ of G1 (source) with $f=0.3 \mathrm{~Hz}$, amplitude $0.1 \mathrm{pu}$.

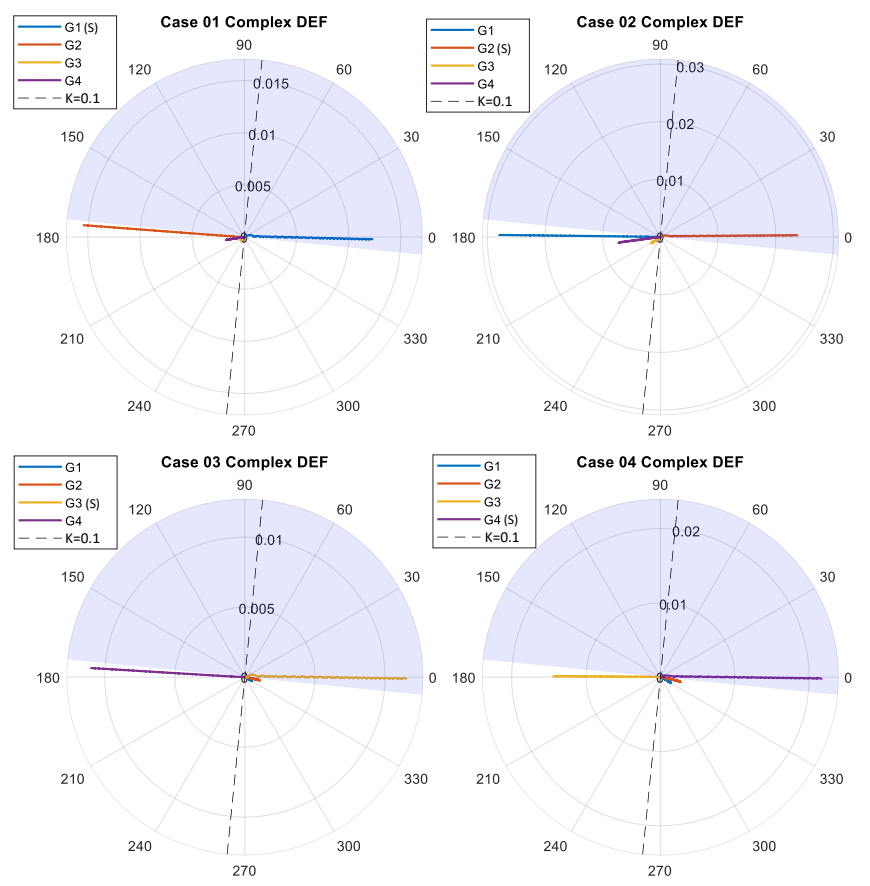

Fig. 2. Complex DEF. Case 1 to 4 . FO in $E_{f d}$. (S) indicates the source.

Additionally, simulations have been carried out by injecting FO into the mechanical power of each generator, one at a time, with a frequency of $0.3 \mathrm{~Hz}$ and an amplitude of $0.1 \mathrm{pu}$ (case 5 to case 8). In these cases, the results provided by the original DEF and the PDEF correctly identify the source. Fig. 3 shows the CDEF for this cases, where it is observed that the projection onto the imaginary axis and the projection onto the line $K+j$ present similar results.

\section{B. 240-bus WECC Test System}

Fig. 4 shows the histogram of the $R / X$ ratio of the branches represented in the WECC 240 model [15]. It can be seen that the largest number of branches are around the value $R / X=0.1$ and for most branches it is less than $R / X=0.15$. 


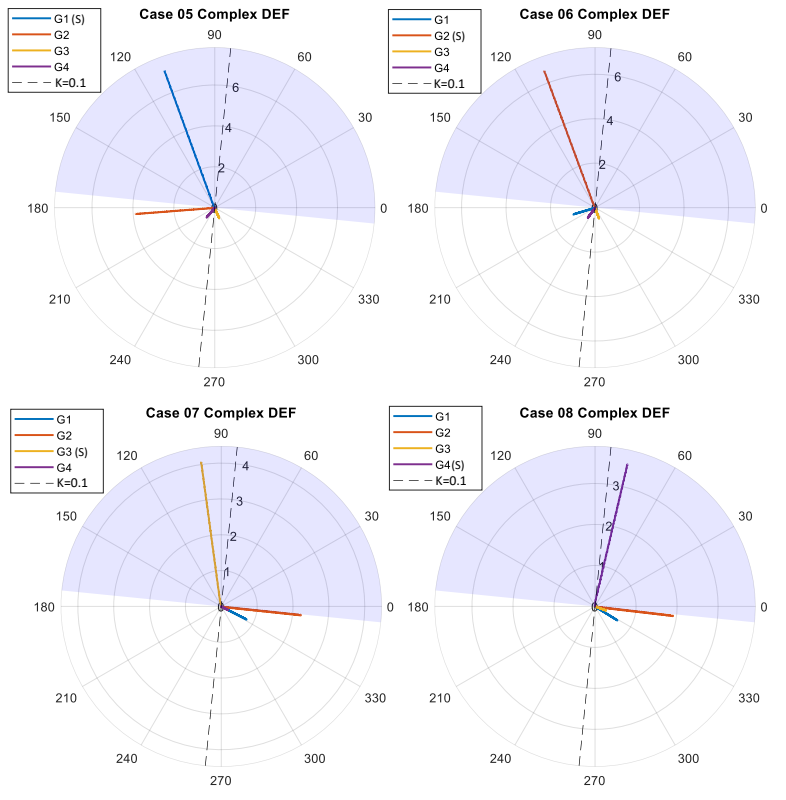

Fig. 3. Complex DEF. Case 05 to 08. FO in Pmech. (S) indicates the source.

In first place, CDEF is applied in one of the cases of IEEENASPI Oscillation Source Location (OSL) Contest [16] where the DEF fails to locate the source of FO. Fig. 4 shows that for case 07 of the OSL Contest the original DEF fails to identify the generator 2634 as a source of FO, while the PDEF with $K=0.1$ manages to identify the generator as a source (positive rate of change of DEF).
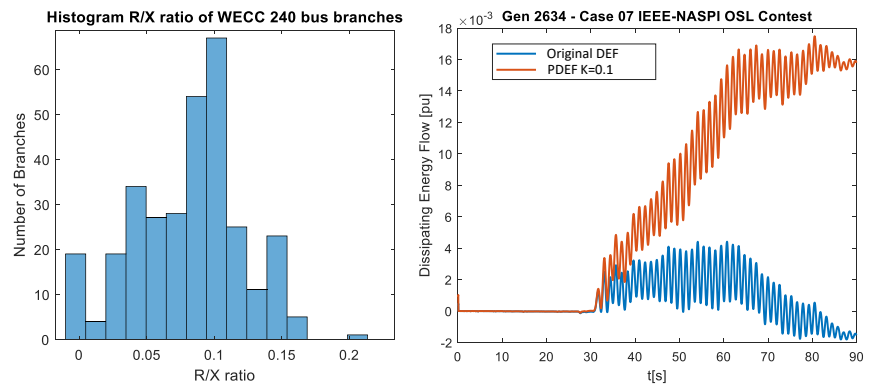

Fig. 4. Histogram of $R / X$ ratio of WECC240 system (left) and dissipating energy flow for Case 07 of IEEE-NASPI OSL Contest (rigth).

Secondly, we reproduce a series of simulations carried out in [4], where the authors identified that the original DEF method has the worst performance when the FO is located in the field voltage of generators, and the FO frequency is the lowest natural frequency of the interarea modes of the system $(0.379$ $\mathrm{Hz}$ ). We apply a FO one at a time in the field voltage and in the mechanical power of the 103 generators that have a excitation system model and are in service. Table I shows a summary of the results on the localization of the plant containing the FO. In cases where the $\mathrm{FO}$ was located in $E_{f d}$, the CDEF improves the performance of the original DEF method from $61.2 \%$ to $91.3 \%(K=0.1)$ and $95.1 \%(K=0.15)$, when the frequency of the FO is $0.379 \mathrm{~Hz}$. In the case of FO located in Governor, both methods perform well.
TABLE I

WECC 240. RESULTS ON PLANT LOCALIZATION CONTAINING FO

\begin{tabular}{|c|c|c|c|c|c|c|c|c|}
\cline { 4 - 9 } \multicolumn{2}{c|}{} & \multicolumn{2}{c|}{ Original DEF } & \multicolumn{2}{c|}{ PDEF K=0.1 } & \multicolumn{2}{c|}{ PDEF K=0.15 } \\
\hline $\begin{array}{c}\text { Location } \\
\text { of FO }\end{array}$ & $\begin{array}{c}\text { Frequency } \\
\text { of FO }[\mathrm{Hz}]\end{array}$ & $\begin{array}{c}\text { Total } \\
\text { cases }\end{array}$ & $\begin{array}{c}\text { Correct } \\
\text { identifi- } \\
\text { cation }\end{array}$ & $\begin{array}{c}\text { Success } \\
\text { rate }\end{array}$ & $\begin{array}{c}\text { Correct } \\
\text { identifi- } \\
\text { cation }\end{array}$ & $\begin{array}{c}\text { Success } \\
\text { rate }\end{array}$ & $\begin{array}{c}\text { Correct } \\
\text { identifi- } \\
\text { cation }\end{array}$ & $\begin{array}{c}\text { Success } \\
\text { rate }\end{array}$ \\
\hline \multirow{2}{*}{ Exciter } & 0.379 & 103 & 63 & $61.2 \%$ & 94 & $91.3 \%$ & 98 & $95.1 \%$ \\
\cline { 2 - 9 } & 0.614 & 103 & 101 & $98.1 \%$ & 103 & $100 \%$ & 103 & $100 \%$ \\
\hline \multirow{2}{*}{ Governor } & 0.379 & 103 & 103 & $100 \%$ & 103 & $100 \%$ & 103 & $100 \%$ \\
\cline { 2 - 9 } & 0.614 & 103 & 103 & $100 \%$ & 103 & $100 \%$ & 103 & $100 \%$ \\
\hline
\end{tabular}

\section{CONCLuSions}

In this paper we propose an extended definition of the DEF that allows an improvement in the performance of the original DEF in cases where it failed. In particular, this improvement is more notorious in cases where the FO source is located in the excitation system of a synchronous generator.

\section{REFERENCES}

[1] L. Chen, Y. Min, and W. Hu, "An energy-based method for location of power system oscillation source," IEEE Trans. on Power Syst., vol. 28, no. 2, pp. 828-836, May 2013.

[2] N. Tsolas, A. Arapostathis, and P. Varaiya, "A structure preserving energy function for power system transient stability analysis," IEEE Trans. on Circuits and Systems, vol. 32, no. 10, pp. 1041-1049, 1985.

[3] Y. Zhi and V. Venkatasubramanian, "Analysis of energy flow method for oscillation source location," IEEE Transactions on Power Systems, vol. 36, no. 2, pp. 1338-1349, 2021.

[4] S. Maslennikov, "Efficiency of the DEF method for locating the source of oscillation," in WECC Oscillations Analysis Work Group, March 2021. [Online]. Available: https://www.wecc.org/Administrative/06_ Maslennikov DEF Efficiency for source locating March\%202021.pdf

[5] Y.-H. Moon, B.-H. Cho, Y.-H. Lee, and H.-J. Kook, "Derivation of energy conservation law by complex line integral for the direct energy method of power system stability," in 38th IEEE Conference on Decision and Control. IEEE. [Online]. Available: https://doi.org/10.1109\%2Fcdc.1999.833278

[6] Y.-H. Moon, H.-S. Ryu, B.-H. Cho, and B. Kim, "Second-kind energy function of power system and its applications," in 2001 Power Engineering Society Summer Meeting, vol. 3, 2001, pp. 1542-1547 vol.3.

[7] Y.-H. Moon, B.-H. Cho, T.-H. Rho, and B.-K. Choi, "The development of equivalent system technique for deriving an energy function reflecting transfer conductances," IEEE Trans. on Power Systems, vol. 14, no. 4, pp. 1335-1341, 1999

[8] K. R. Padiyar, "On the nature of power flow equations in security analysis," Electric Machines \& Power Systems, vol. 9, no. 4-5, pp. 297306, 1984.

[9] K. R. Padiyar and K. K. Ghosh, "A new structure preserving energy function incorporating transmission line resistances," Electric Machines \& Power Systems, vol. 14, no. 3-4, pp. 213-230, 1988.

[10] Y.-H. Moon, B.-H. Cho, Y.-H. Lee, and H.-S. Hong, "Energy conservation law and its application for the direct energy method of power system stability," in IEEE PES 1999 Winter Meeting, 1999, pp. 695-700.

[11] S. Maslennikov and E. Litvinov, "ISO new england experience in locating the source of oscillations online," IEEE Trans. on Power Syst., vol. 36, no. 1, pp. 495-503, 2021.

[12] S. Maslennikov, B. Wang, and E. Litvinov, "Dissipating energy flow method for locating the source of sustained oscillations," Int. J. Elec. Power, vol. 88, pp. 55 - 62, 2017.

[13] P. Kundur, "Power system stability and control," 1994.

[14] Illinois Center for a Smarter Electric Grid (ICSEG). (2021) Kundur's Two Area System Model Data. [Online]. Available: https://icseg.iti.illinois.edu/two-area-system-2/

[15] NREL Test Case Repository. (2021) 240-bus WECC Test System. [Online]. Available: https://www.nrel.gov/grid/test-case-repository.html

[16] (July 2021) IEEE-NASPI Oscillation Source Location Contest. [Online]. Available: http://web.eecs.utk.edu/ kaisun/Oscillation/2021Contest/ 